\title{
Computer Assisted Determination of Mandibular Cystic Lesion Volume from Computed Tomographic Data
}

\author{
Tamer M. Nassef \\ Computer and Programming Engineering Dept. \\ Misr University for Science and Technology, MUST \\ $6^{\text {th }}$ of October City, Egypt \\ tamer.nassef@k-space.org \\ Nahed H. Solouma \\ National Institute of Laser Science \\ Cairo University \\ Giza, Egypt
}

\author{
Reham M. Fliefel, Mona K. Marei \\ Tissue Engineering Lab. \\ Alexandria University \\ Alexandria, Egypt \\ Yasser M. Kadah \\ Biomedical Engineering Dept. \\ Cairo University \\ Giza, Egypt
}

\begin{abstract}
In this paper, a new approach for computing different anatomical regions in dental Computed Tomography (CT) is presented. The approach consists of two steps. First, a HU threshold window sets to separate between different regions upon their gray-level values; second, a set of objects are generated and texture descriptors are calculated for selected windows from the image data sample. Finally, identification of different anatomical regions set for mandible bones to determine the cystic lesion volume based on numerical methods. Preliminary results obtained for dental CT of female patient aged 14 years old complaining of bilateral swelling in her mandible are presented.
\end{abstract}

Keywords-cyctic lesion; tissue engineering; $3 D$ reconstruction; mandible bone simulation; computed tomography

\section{INTRODUCTION}

The term 'cyst' is derived from the Greek word 'kystis' meaning sac or bladder [1]. A cyst may be found in the bone or soft tissue, with an outer wall of connective tissue and an inner wall composed of epithelium most commonly stratified squamous epithelium [2]. Odontogenic cysts are the most common form of cystic lesions that affect the maxillofacial region [3], by definition, these are cysts (i.e. pathological cavities with fluid or semi-fluid contents but excluding pus) with an epithelial lining that derives from the tooth forming organ epithelia, the so-called glands of Serres (rests of the dental lamina), the rests of Malassez (rests of the root sheath of Hertwig) and the reduced enamel epithelium (remnants of the enamel organ after dental crown formation) - although for odontogenic keratocysts it has also been proposed that the lining may derive from mucosal basal cells. In terms of their incidence, radicular cysts are the commonest (mostly associated to teeth with pulp necrosis due to advanced dental caries), followed by dentigerous and odontogenic keratocysts (OKs) [4]. Separate to epithelial-lined cysts, some cystic lesions do not have epithelial linings including mucous extravasation cyst of the salivary glands, the aneurysmal bone cyst and the solitary bone cyst. Despite these examples, most pathologists prefer to describe those pathological cavities not lined by epithelium as 'pseudocysts' [5]. Reichart and Philipsen describe these as 'cavities' rather than cysts; hence, for example, 'aneurysmal bone cavity' [6].

Classifications of cysts are constructed by selecting certain features which are common to a number of conditions and grouping them together on the basis of their shared properties. Either clinical features, histological appearances, concepts of embryological development or the tissue of origin may form the basis of classification or indeed combinations of these considerations [7]. The main factors responsible for cyst development include (in varying degree) [8], proliferation of the epithelial lining and connective tissue capsule, accumulation of fluid within the cyst, and resorption of the surrounding bone and incomplete compensatory repair.

The importance of the correct diagnosis of odontogenic cysts is that some variants, i.e., dentigerous cysts, odontogenic keratocysts, and botroid cysts are aggressive lesions, and it is necessary to distinguish them clinically, radiographically and microscopically from other tumors (such as ameloblastoma and myxoma) so that correct surgical treatment is applied and adequate follow-up instituted [9], It is well known the importance of X-ray diagnostic for the surgical treatment of mandibular cysts. The clinical practice shows that the conventional X-ray methods (extra-oral and intra-oral) ensure enough information concerning removal of the cyst when they are single and small in size. Oblique projections of the left and right mandible, orthopan-tomography and occlusal radiographs for the cortical plates would give precise information for the cyst and its relationship with the surrounding bone and structures. 
Image scanners devices such as computed tomography (CT), magnetic resonance imaging (MRI) or positron emission tomography (PET) are nowadays a standard instrument for diagnosis. Among these devices, CT-scanners are today widely used at radiotherapy departments all over the world it have several advantages. The main advantages of a CT-scanner are to obtain physical information, like patient anatomy, size, shape, and in homogeneities; the other is to obtain the electron density from the different anatomical structures of the patient for the radiotherapy treatment planning [10]. CT examination is not a routine method. It is used to show more precisely the condition and the thickness of the bone plates of the jaw in different levels of the bone. It is fact that patients submit CT examination directly without using the possibilities of conventional methods [11] allow reconstructing threedimensional (3D) images that improve the diagnosis and monitoring of treatments. Axial slices obtained from CT are sent to an independent workstation, which utilizes an appropriate hardware and software to generate 3D images. Those images can be processed by means of different protocols - the 3D surface- and the 3D volume-rendering techniques. The surface-rendering of 3D images allows the assessment of the surfaces of anatomic structures [12].

The treatment of choice is dependent on the size of the lesion, the bony integrity of the cyst and its proximity to anatomical structures (teeth, mandibular canal and maxillary antrum) that may be damaged during surgery [13]. Epithelial odontogenic cysts of the mandible are usually treated by either cystectomy (Enucleation) or cystostomy (Marsipulization). Cysts less than $3 \mathrm{~cm}$ in diameter can be treated by primary excision. Normally, spontaneous bone regeneration results in filling of the residual cavity in the case of small cysts. Osteogenesis of the bony defect in the jaws begins with the formation of a blood clot, which is later replaced by osteogenic granulation tissue in a small defect. The blood clot is replaced by immature bone within a few weeks. This process is completed by osteoblasts which differentiate from the endosteum or marrow spaces, with little or no part being played by the periosteum. Treatment of large lesions, exceeding $3 \mathrm{~cm}$ in diameter, however, is less clear, controversy exists about the treatment of large lesions (ie, spontaneous bone regeneration versus grafting with autogenously grafts, allo-grafts, or combinations of autogenous and allo-plastic materials). Larger bony defects may fill in from the periphery over a period of many months. Incomplete osteogenesis may be seen and such defects may fill with scar tissue partially or completely [14-16]. Cell based therapies for regeneration of bone is now used, in which cells with osteogenic potential are transferred directly to the site requiring augmentation. Because cell based approaches do not depend on local osteoprogenitors for the synthesis of new bone at the site of the defect, they are particularly attractive for patients in whom the host tissue bed has been compromised [17] - [20].

\section{MATERIALS AND METHODS}

A female patient aged 14 years old complaining of bilateral swelling in her mandible. On clinical examination, a firm swelling was found bilaterally in the premolar area. The mucosa was intact and of normal color. The $1^{\text {st }}$ premolar was present on both sides while the Es was also present while the $2^{\text {nd }}$ premolars hadn't erupted yet. On Panoramic radiographic examination, well defined radio fluency was present surrounding the impacted lower $2^{\text {nd }}$ premolars. On aspiration, a straw colored fluid was found having shimmering effect in light.

\section{A. Hounsfield Unite and Gray-Level}

A 3D CT was done to determine the volume of the cystic lesion based on Hounsfield Unite range as in (1).

$$
H U=1000\left(\frac{\mu-\mu_{\text {water }}}{\mu_{\text {water }}}\right) .
$$

Here $\mu$ is the linear attenuation coefficient for the respective material. The linear attenuation coefficient depends on parameters such as electron density, atomic number and the beam quality of the CT-scanner. In the implementation of the Hounsfield scale in this study the Hounsfield scale stretches between $\mathrm{HU}=-1024$ and $\mathrm{HU}=3071$. A HU of -992 represents air outside the patient and a HU of larger than 2832 represents iron. DICOM format files carrying all information about CT scanner, different tissues segmented based on HU range by verified windows defining the tissues pixel positions, each slice has different HU range and this helps in applying the threshold technique to evaluate the difference in bone density for different bone types, this helps to extract bones from slices according to its HU range. To get the parallel gray-level (GL) values from $\mathrm{HU}$ values (2) are used.

$$
G L=\frac{n \times m}{H U_{\text {Range }}} .
$$

Where $n \times m$ are refer to the pixel size of the CT-scanner.

\section{B. Multi-Object Reconstruction Phase}

The multi-object reconstruction helps to extract the mandible bone from skull; that the model will have specific information about mandible, proposed technique is used to extract it from the CT slices, which is implemented with MATLAB program. Triangulation process method is used to generate the $2.5 \mathrm{D}$ models and the marching cubes for the final 3D model; this is applied for all slices as shown in Fig. 1. The external then internal outlines of successive CT scans of a mandible were digitized and linked. The linked outlines were converted into a solid texture. Intensity can be measured by calculating the pixels values multiplied by pixels numbers (3), Table I.

$$
\operatorname{Intensity}(x)=\sum_{i=1}^{N}\left(P x_{n} \times P x_{v}\right) .
$$

Here $\mathrm{Px}_{\mathrm{n}}$ refers to pixel position and $\mathrm{Px}_{\mathrm{v}}$ refers to pixel value and $i$ refers to pixel number. Horizontal and vertical lines, were taken with equal distances to cover the mandible ROI as shown at Fig. 2 ; then the calculation of HU range corresponding to each line are taken place versus distance at $\mathrm{mm}$, shall be repeated to the same process for all slices. 


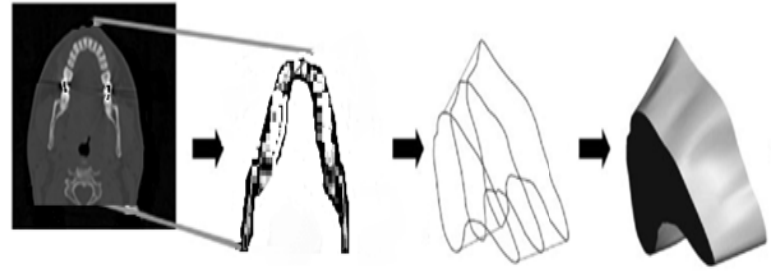

Figure 1. An illustrative example of creating a multi-object model.

TABLE I. INTENSITY MEASURMENTS OF CYST CAVITY

\begin{tabular}{|c|c|c|c|}
\hline $\begin{array}{c}\text { Plane } \\
\text { Number }\end{array}$ & $\begin{array}{c}\text { Number } \\
\text { of Pixels }\end{array}$ & Center (X,Y,Z) & $\begin{array}{c}\text { Total } \\
\text { Intensity }\end{array}$ \\
\hline 13 & 31 & $298.14,218.23,13.0$ & 81045 \\
\hline 14 & 118 & $275.32,214.21,14.0$ & 320484 \\
\hline 15 & 298 & $274.24,211.10,15.0$ & 815393 \\
\hline 16 & 582 & $274.29,207.78,16.0$ & 1594598 \\
\hline & & & \\
$\vdots$ & $\vdots$ & $\vdots$ & $\vdots$ \\
& & & 1631745 \\
\hline 42 & 606 & $263.28,270.21,42.0$ & \\
\hline 43 & 272 & $241.72,268.87,43.0$ & 734706 \\
\hline All & 72113 & $266.33,230.95,29.67$ & 218144702 \\
\hline
\end{tabular}
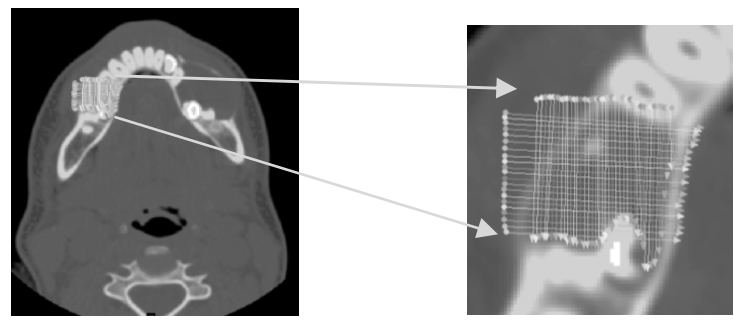

Figure 2. Horizontal and vertical lines at left cyst ROI slice.

\section{RESULTS}

All curves have been compiled horizontal and vertical, as shown at Fig. 3 based on MATLAB code, and to compile the different distances to determine the total distance in $\mathrm{mm}$ for one CT slice (4),(5) are used.

$$
\begin{gathered}
D_{v}=\sum_{i=1}^{L}\left(C b_{v}-C t_{v}\right)-\left(P_{v}+T_{v}\right) . \\
D_{h}=\sum_{i=1}^{L}\left(C l_{h}-C r_{h}\right)-\left(P_{h}+T_{h}\right) .
\end{gathered}
$$

Here $D_{v}, D_{h}$ are refers to vertical and horizontal distance. $L$ equal to line number and $\mathrm{Cb}_{\mathrm{v}}, \mathrm{Ct}_{\mathrm{v}}$ equivalent to cortical bone at both sides bottom and top for the vertical lines, on other hand $\mathrm{Cl}_{\mathrm{h}}$ and $\mathrm{Cr}_{\mathrm{h}}$ are the cortical bone for the other left and right sides for the horizontal lines. $T_{v}$ and $T_{h}$ refers to tooth at vertical and horizontal and finally $\mathrm{P}_{\mathrm{v}}$ and $\mathrm{P}_{\mathrm{h}}$ equal to the undesired bone. To evaluate the total cystic lesion area (6), (7) are used and $\mathrm{D}_{\mathrm{s}}$ or cystic distance around all $\mathrm{S}$ or slices are determined and finally A or cystic area are evaluated.

$$
\begin{aligned}
& D_{s}=\sum_{i=1}^{D_{h}} D_{v} . \\
& A=\sum_{i=1}^{S} D_{s} .
\end{aligned}
$$

Finally the volume of cystic lesion are determined for both sides of mandible by integrated the cystic areas for all slices and added the thickness between slices to evaluate the accurate volume Fig. 4 shows the volume of lift mandibular cystic "6.383 $\mathrm{cm}^{3 "}$.
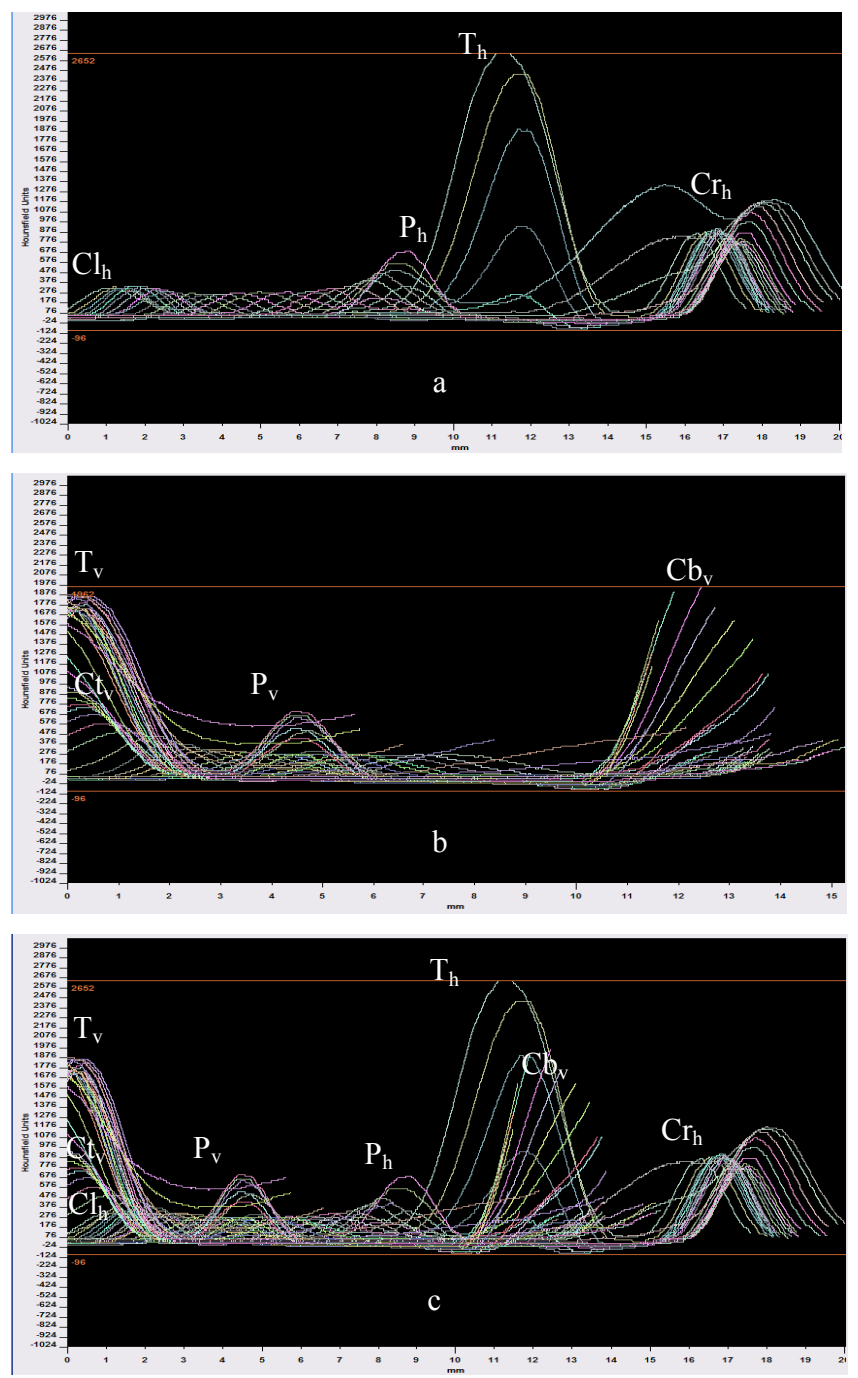

Figure 3. Plotted curves of a) horizontal lines, b) vertical lines and c) both lines at left cyst ROI slice. 

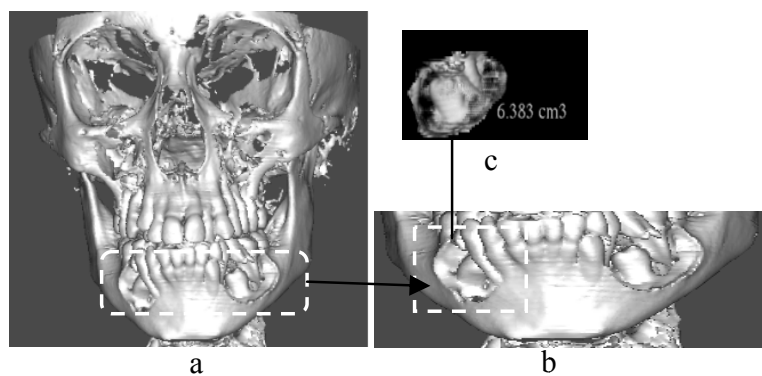

Figure 4. Cystic volume, (a, b) 3D Model of skull with cystic lesion, and (c) the left cystic lesion volume.

\section{CONCLUSION}

CT examination is not a routine method to improve the diagnosis and monitoring treatments for the cyst and its relationship with the surrounding bone and structures, so allow reconstructing three-dimensional (3D) images that given precise information about bone thickness surrounding the cyst are useful, by using different protocols - the 3D surface- and the 3D volume-rendering techniques, this allows the assessment of the surfaces of anatomic structures. This study provide a new approach for computing different anatomical regions in mandible by creating a 3D model based on computed tomography 2D images to assist for determination the cystic lesion volume, some numerical methods are used, pixels intensity values and pixels numbers are significant to verify the volumetric $3 \mathrm{D}$ model of objects, this model can helps in dental diagnosing and to evaluate the orthogonal mechanical properties values especially for edentulous patients.

\section{REFERENCES}

[1] Y. Emes, I. Aktas, B. Atalay, B. Aybar, H. Isseyer, and S. Yalcin, "Bone Healing Following Cyst Enucleation without the Aid of Graft Materials: A Computed Radiographic Analysis," The online Dentistry J. APOLONIA, vol. 20, pp. 65-74, 2008.

[2] K. Sokler, S. Sandy, and J. Grgurevic, "Surgical Treatment of Large Mandibular Cysts,” J. Acta Stomat Croat, vol. 35, pp. 253-257, 2001.

[3] B.G. Koseoglu, B. Atalay, and M.A. Erdem, "Odontogenic Cysts: a Clinical Study of 90 Cases,” J. Oral Science, vol. 46, pp. 253-257, 2004.

[4] G. Landini, "Quantitative Analysis of the Epithelial Lining Architecture in Radicular Cysts and Odontogenic Keratocysts," J. Head \& Face Medicine, vol. 2, pp. 4-9, 2006.

[5] M. Shear, P.M. Speight, "Cysts of the Oral and Maxillofacial Regions," Blackwell Publishing Ltd, Oxford; Ames, Iowa, 2007.

[6] P.A. Reichart and H.P. Philipsen, "Odontogenic Tumors and Allied Lesions, " Lavoisier, Franc, 2004.

[7] H.C. Killey, L.W. Kay, and G.R. Seward, "Benign Cystic Lesions of the Jaws, their Diagnosis and Treatment, " Churchill livingstone, Edinburgh \& London, 1972 .

[8] R. A. Cawson, J. D. Langdon, and J. W. Eveson, "Surgical Pathology of the Mouth and Jaw, " John Wright Publishers, Oxford, 2000.

[9] C.L. Montes, G.C.H. Guerrero, and M.G. Ortíz, "Clinico-Pathologic Study of Odontogenic Cysts in a Mexican Sample Population," J. Arch Med Res, vol. 31(4), pp. 373-376, 2000.

[10] H.Y. Shum, S.C. Chan, and S.B. Kang, "Image-Based Rendering," Springer Science-Business Media, LLC, Spring Street, New York, USA 2007.
[11] H. Mihailova, V. Nikolov, and S. Slavkov, "Diagnostic Imaging of Dentigerous Cysts of the Mandible,” J. IMAB, vol. 8, pp. 8-10, 2008

[12] M.G. Cavalcanti, and J.F. Antunes, "3D-CT Imaging Processing for Qualitative and Quantitative Analysis of Maxillofacial Cysts and Tumors,” J. Pesqui Odontol Bras, vol. 16, pp. 189-194, 2002.

[13] L. Bodner, Y. Woldenberg, and J. Bar-Ziv, "Radiographic Features of Large Cystic Lesions of the Jaws in Children," J. Pediatr Radiol, vol. 33 , pp. 3-6, 2003.

[14] G. Enislidis, N. Fock, I. Sulzbacher, and R. Ewers, "Conservative Treatment of Large Cystic Lesions of the Mandible: A Prospective Study of the Effect of Decompression," B. J. Oral and Maxillofacial Surgery, vol. 42 , pp. 546-550, 2004.

[15] M. Chiapasco, A. Rossi, J.J. Motta, and M. Crescentini, "Spontaneous Bone Regeneration after Enucleation of Large Mandibular Cysts: A Radiographic Computed Analysis of 27 Consecutive Cases," J. Oral Maxillofacial Surgery, vol. 58, pp. 942-948, 2000.

[16] L. Bodner, "Osseous Regeneration in the Jaws Using Demineralized Allogenic Bone Implants," J. Cranio Maxillofacial Surgery, vol. 26, pp. $116-120,1998$.

[17] D. Egrise, D. Martin, A. Vienne, P. Neve, and A. Schouten, "The Number of Fibroblastic Colonies Formed from Bone Marrow is Decreased and the in Vitro Proliferation Rate of Trabecular Bone Cells Increased in Aged Rats," J. Bone, vol. 13, pp. 355-361, 1992.

[18] K. Inoue, H. Ohgushi, T. Yoshikawa, M. Okumura, T. Sempuku, S. Tamai, and Y. Dohi, "The Effect of Aging on Bone Formation in Porous Hydroxyapatite: Biochemical and Histological Analysis," J. Bone Mineral Research, vol. 12, pp. 989-994, 1997

[19] R. Quarto, D. Thomas, and T. Liang, "Bone Progenitor Cell Deficits and the Age-Associated Decline in Bone Repair Capacity," Calcified Tissue Int, vol. 56, pp. 123-129, 1995.

[20] C. Tabuchi, D.J. Simmon, A. Fausto, J.E. Russell, I. Binderman, and L. V. Avioli, "Bone deficit in ovariectomized rats," J. Clin. Invest., vol. 78, pp. 637-642, 1986. 